\title{
Poor ovarian reserve and male infertility in a rural in vitro fertilization setup: a case report
}

\author{
Neha V. Harne*, Vaibhav K. Nadkarni, Purnima Nadkarni, Jigna Garasia
}

Nadkarni Hospital and Test Tube Baby Centre, Killa Pardi, Valsad, Gujarat, India

Received: 30 July 2021

Revised: 04 September 2021

Accepted: 06 September 2021

\section{*Correspondence:}

Dr. Neha V. Harne,

E-mail: nehaharne081192@gmail.com

Copyright: (c) the author(s), publisher and licensee Medip Academy. This is an open-access article distributed under the terms of the Creative Commons Attribution Non-Commercial License, which permits unrestricted non-commercial use, distribution, and reproduction in any medium, provided the original work is properly cited.

\begin{abstract}
Female fertility begins to decline many years prior to the onset of menopause despite continued regular ovulatory cycles. Although there is no strict definition of advanced reproductive age in women, infertility becomes more pronounced after the age of 35 . In the female, the number of oocytes decreases with age until the menopause. Oocyte quality also diminishes, due in part to increased aneuploidy because of factors such as changes in spindle integrity. Although older male age affects the likelihood of conception, abnormalities in sperm chromosomes and in some components of the semen analysis are less important than the frequency of intercourse. Age is as accurate as any other predictor of conception with assisted reproductive technology.
\end{abstract}

Keywords: Poor ovarian reserve, Male infertility, ICSI, Diabetes in male

\section{INTRODUCTION}

Since ancient times, humankind has been obsessed with fertility. With both biological and social imperatives driving us to have children, it is no surprise that, even today the lack of fertility is perceived as many as a curse in India, specially the rural areas, this has resulted in many social evils such as stigmatization and isolation, and in turn has caused trauma to many individuals. Thus proper diagnosis of infertility is of paramount important not only to the physical wellbeing but also to the mental and social wellbeing.

Infertility is inability of a sexually active, noncontracepting couple to achieve spontaneous pregnancy within one year.

Ovarian reserve is the adequate follicular development of ovaries in response to gonadotropins. The primary need of ovarian reserve marker is to provide assistance in selection of the right protocol with correct initial dose of gonadotropins for controlled ovarian stimulation in IVF cycle. As a result the markers for ovarian reserve prior to controlled ovarian stimulation are frequently done to predict outcome in IVF cycles. $^{1}$

The lynchpin of the diagnosis of infertility in men, semen analysis forms the basis of evaluation of male partner in sub fertile couples.

\section{CASE REPORT}

A 41 years old female patient married for 5 years, a known case of hypothyroidism presented to the OPD as a case of primary subfertility. She had regular menses with average flow. Physical examination was done with a negative Pap smear test.

Baseline investigations revealed AMH levels of $0.3 \mathrm{ng} / \mathrm{ml}$ with basal FSH $13 \mathrm{miu} / \mathrm{ml}$ indicative of diminished ovarian reserve (DOR) and that her biological clock was running out of time. Transvaginal scan showed an AFC of 
3 in the right ovary and 2 in the left. A diagnostic hysteroscopy was done, findings suggestive of thin adhesions and intrauterine polyp. Adhesiolysis and polypectomy was done for the patient and was thereafter treated with sequential estrogen and progesterone therapy along with DHEA supplements for 2 months improve the egg giving capacity.
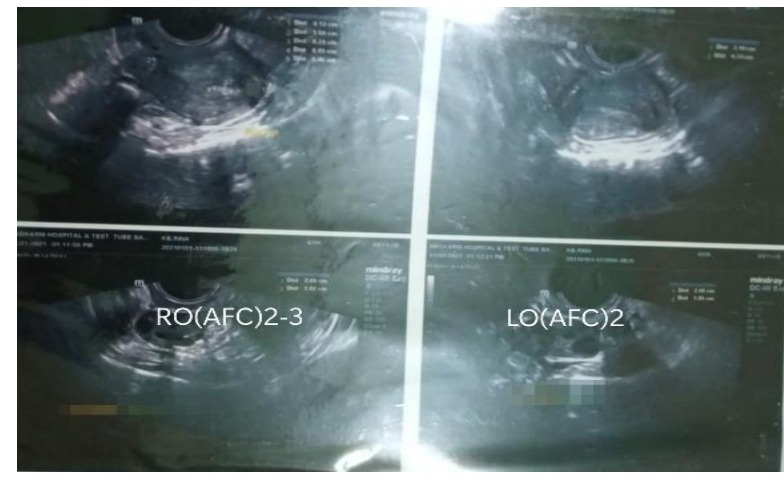

Figure 1: Radiography.

Her husband, a 48 years old male was a known case of poorly controlled type II diabetes mellitus on insulin. He had also undergone renal transplant, since then he was on various drugs like immune suppressants, antihypertensives, steroids and insulin. Semen analysis showed hypospermia $(0.3 \mathrm{ml})$, count of 60 million with $0 \%$ motility. HOS test was done only to increase the motility to $10 \%$. Estradiol level of $<11.80 \mathrm{pg} / \mathrm{ml}$, testosterone levels $365 \mathrm{ng} / \mathrm{ml}$ and FSH of $8.7 \mathrm{miu} / \mathrm{ml}$. After consultation with andrologist he was started on antioxidant. Strict control of blood sugar levels was advised. Semen analysis was repeated after one month which showed volume of $0.4 \mathrm{ml}, 60$ million sperm count and a motility of $20 \%$. Husband sperm freezing was done. Karyotyping of both partners were normal.

As the patient had poor ovarian reserve with male factor infertility, patient was henceforth advised intra cytoplasmic sperm injection (ICSI).

Antagonist protocol was started with recombinant FSH 300 and HMG 75 for 5 days. The dose of HMG was increased to 150 along with addition of growth hormone from day 6. Antagonist was added on day 7.21 oocytes were retrieved, amongst which 10 were MII oocytes. On fertilization 3 embryos were formed ( 2 grade I and 1 grade II). Fresh transfer was postponed due to high progesterone levels.

A second cycle for oocyte retrieval was started using microdose long protocol in which triptorelin acetate 20 units for 5 days in the luteal phase of the previous cycle which was reduced to 10 units from day 2. HMG 300 units added from day 2 of the cycle which was stepped up to 450 units from day 5.17 oocytes were retrieved, amongst which 12 were MII and 5 embryos were formed (3 grade I and 2 were grade II).
Frozen embryo transfer was done sequentially with two day 3 embryos and one blastocyst $(2+1)$. After 15 days beta-HCG was $650 \mathrm{miu} / \mathrm{ml}$ and a second report done 7 days later was $4500 \mathrm{miu} / \mathrm{ml}$.

\section{Analysis}

The patient had combination of two severe factors that impacted the biological clock namely, age and poor ovarian reserve. Both affected the number of available eggs as well as the competency. It should be noted that the quality of egg rather than the sperm quality impacts far more resulting in chromosomal aberration (aneuploidy in embryo). At 41 years of age under the best circumstances only one out of 10 eggs were likely to be chromosomally normal (euploid). It showed poor likelihood of any given embryo being competent to result into a viable pregnancy at such advanced age. Then to make matters worse, a poor ovarian reserve translated into fewer eggs being available to start with. Thus with increasing age and fewer eggs the likelihood of resulting embryos being competent and the chance of successful IVF was not great.

The husband with a systemic disease like diabetes mellitus and end stage nephropathy and renal transplant which affected fertility, hormonal test should be done and was to be evaluated and treated for the same. It undoubtedly added another negative component which further reduced fertilization potential and increased the likelihood of aneuploidy.

\section{Recommendations}

Choosing the optimal protocol for controlled ovarian stimulation

With the use of supplements like DHEA, vitamin D, antioxidants, aspirin for improving ovarian reserve along with selection of the right protocol in optimal doses of gonadotropins was an important factor for successful outcome in such patients with DOR. The use of growth hormone throughout the stimulation in the hope that it enhanced the mitochondrial function and enhancing the oocyte quality.

\section{The male factor}

It was relatively unlikely that with such low motility any specific treatment other that IVF would improve the outcome. However, a full urologic examination with evaluation of varicose vein in the scrotum should be done. Considerations of long term immune suppressants to be thought of as well.

\section{Egg donation option}

IVF using egg donation was the most rationale approach yielding best possible outcome at such advanced age with decreased ovarian reserve. However the couple was adamant to have baby with their own gametes. Given this 
decision, it was essential that every effort to be made to optimize the chance of assessing euploid competent embryos for transfer to the uterus

\section{Pre implantation genetic testing and embryo banking}

Due to the advanced age and the decreased ovarian reserve the couple were advised for blastocyst biopsy for PG testing and that all euploid blastocyst be banked and stockpiled overtime.

Most importantly time was of the essence here.

\section{DISCUSSION}

The chance of a women getting pregnant reduced slowly but significantly over the time beginning around 32 years, but decline was more rapid after 37 years of age. Education and enhanced awareness of the effect of age on fertility were essential in counselling the patient who desired pregnancy. There was expected reduction in fertility related to age, the raised incidence of problems that reduced fertility and the increased risk of spontaneous abortion and aneuploidy in women who were above 35 years. ${ }^{2}$ Other causes of fertility decline in females with age were considered to be due to reduced frequency of intercourse and diseases of uterus owing to increased age or ageing oocytes giving rise to poor quality embryos. ${ }^{2,3}$ Mitochondial dysfunction due to oxidative damage to mitochondria was considered to be the reason behind somatic cell changes due to age. ${ }^{3}$ Prevalence of permanent childlessness was increasing in many women by voluntary postponement of motherhood.

Reduction in fecundity of men with age was modest. Both, men and women will undergo a compounded effect of their separate age related changes. A couples fecundability at age of 40 years would be approximately half of what it was at age 30 years. ${ }^{4}$ It was essential to counsel the fertility, seeking couple that there was reduction of the intrinsic fertility potential of oocyte after 35 years of age which was most important cause of decreasing fertility. ${ }^{5}$ There was an increase in embryo aneuploidy, with increase in age this needed to be stressed to the couple. ${ }^{6}$ Couple may not be able to have desired number of children if they delay childbearing attempts and increase the maternal age may be the absolute risk factor of family size being apparently limited by biological factors. ${ }^{7}$

Couples also appeared to experience declining fecundability with increasing age of the male partner, especially in which the man was older than 40 years. Reduced fertility was observed in attempts at assisted reproduction when the sperm counts from men more than 50 years old. Increased time to pregnancy was seen frequently with increasing age of the male partner which was proved by findings of decline in semen volume, sperm motility and sperm count. ${ }^{4}$ Several other factors associated with increasing age in men and their reproductive health were affected. Factors such as sexual activity, fertility and androgen status reduce significantly and steeply as age of men rises. Germinal epithelium continued to produce male gametes from puberty till death in men which was rarely interrupted by factors like disease. Sexual activity in male decreased rapidly with ageing and the increased instances of sexual dysfunction that were mainly caused by neurovascular defects in the hydraluric mechanism that were essential in erection of penis. ${ }^{8}$ Over the years, significant reduction was seen in semen quality of men. In 1938, sperm concentration used to be around 113 million $/ \mathrm{ml}$, which had reduced to mean average of 66 million/ml. ${ }^{9}$ Such decline in semen quality was being observed and reported uniformly in many parts of the world. It was also proved that increase in sex hormone binding globulin (SHBG) levels reduced the endocrine function by the decrease in testosterone levels. There was reduction of leydig cells, sertoli cells, daily sperm production and various other morphological alterations in ageing tests. ${ }^{10}$ All these findings suggested that the couples fecundity was reduced by rising paternal age.

Diseases such as diabetes mellitus can affect spermatogenesis at various levels. It was one of the most common endocrine disorder. It interfered with sexual function. Both neurological and vascular factors cause erectile dysfunction in diabetes and autonomic neuropathy caused ejaculatory dysfunction. Impotence was very common complication of diabetes and occured in $50 \%$ of patients. Retrograde ejaculation progressed to ejaculatory failure. Testicular biopsies from impotent diabetic patients showed huge range of changes varying from minimal reduction in spermatogenesis to completely hyalinized and non-functional seminiferous tubules. If testis was reduced in size with FSH raised, primary testicular disease was suspected and significant damage to sperm production will be there. Thus diabetic patient was susceptible to develop sperm maturation disorders. If the patient was able to produce good fertile ejaculate it was wise to cryopreserve semen for future insemination because any time primary testicular disease or ejaculatory failure may supervene.

In the backdrop of such declining fertility, the world was seeing surge in people opting for assisted reproductive technology (ART) treatments. Due to increase age, there was tendency to opt for ART treatments like ICSI, pre implantation genetic screening (PDS) and also donor gametes. Successful outcomes of self-fresh cycles were mainly dependent upon the maternal age, and women aged under 30 years (accounting to $38 \%$ ) had highest rate of delivery per embryo transferred. This rate reduced with increasing age of women with only $9.9 \%$ in women of age 40-44 years and further declined to just $1.2 \%$ in women with age 45 years or more. ${ }^{11}$

Awareness about the impact of age on oocyte had brought many women to the decision of freezing their oocytes by cryopreservation technology if they were planning to postpone their pregnancy. 


\section{CONCLUSION}

POR is an indicator of reduced size of primordial follicular pool, and the resulting eggs are likely to be of suboptimal quality as well. An early recourse to IVF remains the only option with reasonable chance of achieving pregnancy in such women. None of the available therapeutic interventions have the ability to overcome the barriers of low quantity and quality of eggs in women with POR. Male infertility is an important cause of infertility with a strong impact on the psychology and physiology of couple. It can be due to several reasons. Therefore, it's the need of the hour to look into the factors which are causing such a rise in male infertility and attempts should be made to control such factors in near future.

\section{Funding: No funding sources}

Conflict of interest: None declared

Ethical approval: Not required

\section{REFERENCES}

1. Maheshwari A, Fowler P, Bhattarcharya S. Assessment of ovarian reserve-should we perform tests of ovarian reserve routinely? Human Reproduct. 2006;21(11):2729-35.

2. ACOG committee on gynaecolgic practice and practice committee. Female age related fertility decline. Committee opinion no. 589. Fertil Steril. 2014;101(3):633-4.

3. Paul S. Is age-related decline in female fertility a mitochondrial dysfunction? Obstet Gynecol Int J. 2016;5(2):00148.

4. Rothman KJ, Wise LA, Sørensen HT, Riis AH, Mikkelsen EM, Hatch EE. Volitional determinants and age-related decline in fecundability: a general population prospective cohort study in Denmark. Fertil Steril. 2013;99(7):1958-64.

5. Silber SJ, Kato K, Aoyama N, Yabuuchi A, Skaletsky $\mathrm{H}$, Fan Y, et al. Intrinsic fertility of human oocytes. Fertil Steril. 2017;107(5):1232-7.

6. Demko ZP, Simon AL, McCoy RC, Petrov DA, Rabinowitz M. Effects of maternal age on euploidy rates in a large cohort of embryos analyzed with 24chromosome single-nucleotide polymorphism-based preimplantation genetic screening. Fertil Steril. 2016;105(5):1307-13.

7. Joffe M, Key J, Best N, Jensen TK, Keiding N. The role of biological fertility in predicting family size. Human Reproduct. 2009;24(8):1999-2006.

8. Handelsman D. Ageing and male reproductive health. Human Reproduct. 2010.

9. Jofee M. What has happened to human fertility? Hum Reproduct. 2010;25(2):295-307.

10. Ford WCL, North K, Taylor H, Farrow A, Hull MGR, Golding $\mathbf{J}$, et al. Increasing paternal age is associated with delayed conception in a large population of fertile couples: evidence for declining fecundity in older men. Human Reproduct. 2000;15(8):1703-8.

11. Fitzgerald O, Paul RC, Harris K, Chambers GM. Assisted reproductive technology in Australia and New Zealand 2016. Sydney: National Perinatal Epidemiology and Statistics Unit (UNSW); 2018.

Cite this article as: Harne NV, Nadkarni VK, Nadkarni P, Garasia J. Poor ovarian reserve and male infertility in a rural in vitro fertilization setup: a case report. Int J Reprod Contracept Obstet Gynecol 2021;10:4020-3. 